\title{
Spatial- and spatio-temporal pattern formation in optically driven discrete systems
}

\author{
Victor M.Burlakov \\ Institute of Spectroscopy Russian Academy of Sciences, 142092 Troitsk, \\ Moscow region, Russia
}

\begin{abstract}
Dynamical coherent structure (pattern) formation in the Klein-Gordon lattice excited by periodic external field near the optical resonance is studied. It is shown that besides spatial patterns discovered recently (V.M.Burlakov, Phys.Rev.Lett. 80, 3988 (1998)) the spatio-temporal patterns can be generated in the lattice in rather broad region of excitation parameters. An influence of cubic anharmonicity in the lattice potential and that of random noise on the pattern formation is examined.
\end{abstract}

\section{INTRODUCTION}

Dynamical coherent structure (pattern) formation is a new phenomenon in dynamics of anharmonic lattices. So far patterns have been studied basically in continuous systems (see Ref.1 and references therein) and recently in granular materials [2 [5]. Some soliton-like localized structures have been studied in driven anharmonic lattices [6 9 . Recently it has been found that optically driven Klein-Gordon (KG) lattice with quartic anharmonicity possesses pattern formation, i.e. formation of a combination of few standing waves, or, in the other words, lattice spatial modes (LSMs), vibrating with one and the same frequency $\omega$ equal to that of the driving field [10]. The important factors for the pattern formation are: i) modulation instability (MI) of the $k=0$ LSM directly excited by external field and resulting in generation of other LSMs composing the pattern; ii) destructive interference of modulation instabilities of different LSMs resulting in the pattern stability. Due to interference of constituent LSMs with each other the pattern looks like a train of equal intrinsic localized modes (ILMs) extensively studied lately 11 16]. Optical creation of a random ensemble of ILMs have been recently proposed by Rössler and Page for model system [17] and by Lai and Sievers for realistic spin wave systems [18]. Due to equality of the constituent ILMs the pattern formation opens a new way of experimental identification of ILMs based on dynamical breaking of the lattice translational symmetry.

To make further step toward experimental identification of patterns (or ILMs) it seems expedient to study the pattern generation and stability in optically driven Klein-Gordon (KG) lattice with both cubic and quartic anharmonicity and in the presence of thermal fluctuations (random noise). In the present paper I show that the pattern formation conditions in the KG lattice with cubic and quartic anharmonicity can be analyzed on a bases of the lattice with pure quartic anharmonicity. The pattern generation and stability regions in the space of external excitation parameters are presented. It is shown that at certain conditions random noise results in frustration of the stationary spatial patterns discovered in [10] giving rise to a new type of patterns. The particles vibration amplitude in the new patterns is modulated in both space and time so one may consider them as spatio-temporal patterns. The latter can be generated close to the borders of the spatial patterns stability regions and probably correspond to a transition regime from spatial patterns to chaos.

\section{MI OF OPTICALLY EXCITED STANDING WAVE IN THE KLEIN-GORDON LATTICE}

At the initial stage of the pattern formation the perturbation LSM with $k_{p}$ is amplified due to MI of the excited $k=0$ nonlinear LSM (referred below as to excited carrier LSM, or excited LSM). Simultaneously the higher spatial harmonics $2 k_{p}, 3 k_{p}$, ets. will be generated due to 3 -wave and/or 4-wave mixing of the excited LSM and the perturbation LSM. Growing up of the higher harmonics, their mutual interaction and coupling to the excited LSM finally lead to the pattern formation. Thus, the MI of the excited LSM works as an ignition for the pattern formation.

Specific feature of the KG lattice is that it possesses a single optical band of vibrations. The MI of free vibrating waves in various anharmonic lattices including the KG one was analyzed in 1925 . Essential points for analysis of MI in the driven lattice are those related to i) optical excitation at frequency $\omega$ which is different from the eigen frequency of the $k=0$ LSM; ii) phenomenological damping which mimic all the dissipative interactions between the phonon band under consideration and any other excitations in realistic system. Note, that the dissipative interactions which involve only the vibrations from the optical band of the KG lattice are rigorously treated in the present analysis.

Motion equation for $n-t h$ particle of unity mass and charge in the KG lattice is 


$$
d^{2} U_{n} / d t^{2}+\gamma \cdot d U_{n} / d t+\omega_{0}^{2} U_{n}+K_{2} \cdot\left(2 U_{n}-U_{n-1}-U_{n+1}\right)+K_{3} U_{n}^{2}+K_{4} U_{n}^{3}=E_{0} e^{i \omega t}+\text { c.c. }
$$

where $\gamma$ is phenomenological damping constant, $\omega_{0}^{2}, K_{4}$ are incite and $K_{2}$ is intercite force constants, $E_{0}$ is the external field amplitude. The trial solution of Eq.(1) for small anharmonicity can be chosen in the form

$$
U_{n}(t)=\frac{1}{2}\left[V_{C 0}+V_{C 1} \exp \left(i \omega t+i \varphi_{1}\right)+V_{C 2} \exp \left(i 2 \omega t+i \varphi_{2}\right)+c . c .\right]
$$

where $V_{C j}$ and $\varphi$ are real amplitude and phase angle respectively. Two types of the KG lattice are considered. The lattice with pure quartic anharmonicity (L1): $\omega_{0}=354, K_{2}=6 \cdot 10^{4}, K_{3}=0, K_{4}=-3.3 \cdot 10^{6}$; the lattice with both cubic and quartic anharmonicity (L2): $\omega_{0}=354, K_{2}=6 \cdot 10^{4}, K_{3}=6 \cdot 10^{5}, K_{4}=5 \cdot 10^{5}$. The incite potential curves for a particle in the L1 and L2 lattices are shown in Fig.1a. A particle vibration in the right potential well is to be considered. One can see from Fig.1a that the particle remains in the minimum until its vibration amplitude do not exceed the value $U_{t h}^{0} \simeq 0.2$. The latter value can be considered as a natural threshold for the point defect formation related to the out-off minimum jump of the particle. The parameters of the L1 and L2 lattices were chosen in a way for the $V_{C 1} \propto E_{0}$ dependences to be nearly the same (see Fig.1b). This choice allows to understand a pure influence of cubic anharmonicity not related to anharmonic shift of the optical mode frequency. For $\omega<\omega_{0}$ the $V_{C j} \propto E_{0}$ dependences show bistability and for $\omega>\omega_{0}$ these dependences are monotonous. Below only the latter case is considered in detail.

Obviously $V_{C 0}=V_{C 2}=0$ in the L1 lattice and one may use the rotating wave approximation (RWA) for stability analysis of the excited LSM. According to Ref. [10] the perturbation growing rate (increment) $\operatorname{Im}(\Omega(q))$ can be determined from the equation

$$
\left[\omega_{q}^{2}-(\omega+\Omega)^{2}-i \gamma \cdot(\omega+\Omega)\right] \cdot\left[\omega_{q}^{2}-(\omega-\Omega)^{2}+i \gamma \cdot(\omega-\Omega)\right]=\beta^{2},
$$

obtained after substitution of (2) with perturbation

$$
\delta U_{n}=\frac{1}{2} \cos (q n) \cdot\left[V_{P 1} \exp (i \cdot(\omega-\Omega) t)+V_{P 2} \exp (i \cdot(-\omega-\Omega) t)+c . c .\right],
$$

into Eq(11) (here and below the wavevector of perturbation LSM is denoted by $q$ while that of carrier LSM is denoted by $k$ ). Here $\omega_{q}^{2}=\omega_{0}^{2}+4 K_{2} \sin (q / 2)^{2}+\frac{3}{2} K_{4} V_{C 1}^{2}, \beta=\frac{3}{4} K_{4} V_{C 1}^{2}, V_{P j}$ is the complex amplitude, and $q$ and $\Omega$ are the wave vector and the complex frequency shift of the perturbation wave respectively.

To study MI of the solution (2) in general case the perturbation has to be chosen in the form

$$
\begin{gathered}
\delta U_{n}=\frac{1}{2} \cos (q n) \cdot\left[V_{P 0} \exp (-i \Omega t)+V_{P 1} \exp (i \cdot(\omega-\Omega) t)+V_{P 2} \exp (i \cdot(-\omega-\Omega) t)+\right. \\
\left.V_{P 3} \exp (i \cdot(2 \omega-\Omega) t)+V_{P 4} \exp (i \cdot(-2 \omega-\Omega) t)+\text { c.c. }\right] .
\end{gathered}
$$

The $\operatorname{Im}(\Omega(q))$ function then was calculated numerically equating to zero the determinant of the corresponding system of five liner equations. Relative increment $\operatorname{Im}(\Omega(q) / \omega)$ for the L1 and L2 lattices and that for free carrier LSM with $k=0$ in the L2 lattice with $\gamma=0$ are plotted in Figs.2a-c respectively for $\omega=1.05 \omega_{0}$ and three values of $V_{C 1}$. One can see that the instability regions $(\operatorname{Im}(\Omega(q))>0)$ in the $q$ space for the excited carrier LSM in the L1 lattice are much broader and the instability is stronger than those in the L2 lattice. Note that the instability of free carrier LSM is much stronger than that of excited one (compare Fig.2b and Fig.2c). By symbols in Fig.2b are shown the $\operatorname{Im}(\Omega(q) / \omega)$ curves for the system with pure quartic anharmonicity which will be used for approximation of the L2 lattice. The effective quartic anharmonicity constant was chosen to be $K_{4}^{e f f}=K_{4} \cdot\left(1+\left(\frac{K_{3}}{\omega_{0}^{2}}\right)^{2} \cdot V_{C 1}^{2}\right)-\frac{11}{12}\left(\frac{K_{3}}{\omega_{0}}\right)^{2}$ and also the factor $\beta$ in (3) was changed in this case to $\beta^{e f f}=\frac{7}{12} K_{4} V_{C 1}^{2}$. The numerical factors $\frac{11}{12}$ and $\frac{7}{12}$ were chosen for the best fit of the increment curves shown by lines in Fig.2b. One can see that the system with the effective quartic anharmonicity approximates rather well the original L2 lattice if the excitation field $E_{0}$ is not very high.

This approximation allows to suitably represent the excited LSM instability regions in the $(E, \gamma)$ space. The MI regions of the excited LSM for the perturbation wave vectors $q=\pi / 3$ and $q=\pi / 2$ are shown in Fig.3 by solid lines. The curves were obtained from Eq.(3) under conditions $\Omega(q=\pi / 3)=0$ and $\Omega(q=\pi / 2)=0$ for the left and right closed regions respectively. Additionally, the excited LSM amplitude must be restricted by a value much lower than the $U_{t h}^{0}$. This is because the vibration amplitude of some particles in the pattern increases compared to its value in the excited LSM and may result in the out-of minimum jump of these particles. In the case shown in Fig. 3 the excited LSM amplitude was restricted by the value $0.13 \cdot a$, i.e. about $0.7 \cdot U_{t h}^{0}$, what cuts the MI regions for high $E$ values. In fact, the difference between particles vibration amplitudes in the excited LSM on one hand and in the pattern on the other hand depends on damping so the MI regions can be more extended to the higher $E$ values at high $\gamma$ values. 


\section{PATTERN SOLUTIONS FOR $K_{3}=0$ AND THEIR STABILITY}

The perfect pattern can be generated starting from the seeding LSM $\sim \cos (q \cdot n)$ at $t=0$. Then under action of external field $E=E_{0} e^{i \omega t}$ the system will pass through two stages: a) excitation of carrier LSM; b) growing up of the seeding LSM due to MI of the excited carrier LSM and simultaneous generation of other LSMs due to four-wave mixing. Obviously, if the number of generated LSMs at the initial stage of the pattern formation is fairly restricted $(3 \div 5)$ the LSMs may grow up to large enough amplitude to be as additional nonlinear waves (carrier LSMs) in the lattice and to stabilize the pattern. We restrict our consideration by the total number of carrier LSMs $N_{L S M}=3$ and $N_{L S M}=4$ (3-LSM- and 4-LSM patterns, respectively). The former is build up of the carrier LSMs with wave vectors $k_{1}=0, k_{2}=\pi / 2$ and $k_{3}=\pi$ (lattice constant $a=1$ ) while the latter with those $k_{1}=0, k_{2}=\pi / 3, k_{3}=2 \pi / 3$ and $k_{4}=\pi$. The MI of the excited carrier LSM must have a maximum around $q_{\max }=\pi / 2$ in case of $N_{L S M}=3$ and around $q_{\max }=\pi / 3$ for $N_{L S M}=4$. This is the condition for $E_{0}$ or, in the other words, for the excited carrier LSM amplitude $V_{C}$. For $N_{L S M}=2\left(q_{\max }=\pi\right)$ no solutions have been found.

Because of the symmetry arguments the pattern may consist of standing LSMs only. The trial pattern solutions are

$$
\begin{gathered}
U_{n}^{3-L S M}(t)=\frac{1}{2}\left[V_{C 1} \exp \left(i \omega t+i \varphi_{1}\right)+V_{C 2} \cos \left(\frac{\pi}{2} n\right) \exp \left(i \omega t+i \varphi_{2}\right)+\right. \\
\left.V_{C 3} \cos (\pi n) \exp \left(i \omega t+i \varphi_{3}\right)+c . c .\right], \\
U_{n}^{4-L S M}(t)=\frac{1}{2}\left[V_{C 1} \exp \left(i \omega t+i \varphi_{1}\right)+V_{C 2} \cos \left(\frac{\pi}{3} n\right) \exp \left(i \omega t+i \varphi_{2}\right)+\right. \\
\left.V_{C 3} \cos \left(\frac{2}{3} \pi n\right) \exp \left(i \omega t+i \varphi_{3}\right)+V_{C 4} \cos (\pi n) \exp \left(i \omega t+i \varphi_{4}\right)+c . c .\right],
\end{gathered}
$$

for 3-LSM and 4-LSM patterns respectively. No new LSMs important within RWA will appear due to four-wave mixing of those chosen. Here again $V_{C j}$ are real amplitudes and $\varphi_{j}$ are phase angles. According to $\omega$ and $\gamma$ values a single stable nontrivial (all $V_{C j} \neq 0$ ) solution $\Phi_{1}$ of the form (6)-(6) was found in the region between the dotted curves in Fig.3. Outside this region the solution $\Phi_{1}$ is unstable in the sense discussed below. An additional and strongly unstable solution $\Phi_{2}$ [10] exists for the 3-LSM pattern at $\gamma<0.1 \cdot \omega_{0}$ while for 4-LSM patterns no additional solutions have been found. The examples of the 3- and 4-LSM patterns of the $\Phi_{1}$-type calculated after substitution of (6) and (7) respectively into Eq.(11) with the parameters corresponding to the points $\mathbf{a}$ and $\mathbf{c}$ in Fig.3 are presented in Fig.4. Note, that indeed the patterns can be regarded as the lattices of intrinsic localized vibrations of the odd parity [11, 12].

Linear stability analysis of the solution (6) within RWA was given in [10]. Similar approach to the 4-LSM pattern stability requires the total perturbation to contain all perturbation waves coupled to each other via four-wave mixing, i.e. all spatial harmonics resulting from a product of any three carrier LSMs from Eq.(7) on a perturbation wave. One can see that a set of waves

$$
\begin{aligned}
& \delta U_{n}= \frac{1}{2}\left\{\operatorname { e x p } ( i \cdot ( \omega - \Omega ) t ) \cdot \left[V_{P 1} \cos (q n)+V_{P 3} \cos \left(\left(\frac{\pi}{3}-q\right) n\right)+V_{P 5} \cos \left(\left(\frac{\pi}{3}+q\right) n\right)+\right.\right. \\
&\left.V_{P 7} \cos \left(\left(\frac{2 \pi}{3}-q\right) n\right)+V_{P 9} \cos \left(\left(\frac{2 \pi}{3}+q\right) n\right)+V_{P 11} \cos ((\pi-q) n)\right]+ \\
& \exp (i \cdot(-\omega-\Omega) t) \cdot\left[V_{P 2} \cos (q n)+V_{P 4} \cos \left(\left(\frac{\pi}{3}-q\right) n\right)+V_{P 6} \cos \left(\left(\frac{\pi}{3}+q\right) n\right)\right. \\
&\left.\left.+V_{P 8} \cos \left(\left(\frac{2 \pi}{3}-q\right) n\right)+V_{P 10} \cos \left(\left(\frac{2 \pi}{3}+q\right) n\right)+V_{P 12} \cos ((\pi-q) n)+c . c .\right]\right\}
\end{aligned}
$$

fulfills this condition. Indeed the set of waves (8) contains spatial harmonics $k_{p}= \pm q+\frac{\pi}{3} m(m=0,1,2,3)$. After coupling to any three carrier LSMs from (7) it results in a set of spatial harmonics with wave vectors $k_{p}= \pm q+\frac{\pi}{3} m \pm \frac{\pi}{3} l$ $(l=0,1,2,3)$ which obviously can be reduced to (8). System of twelve linear equations derived after substitution of (7) and (8) into Eq.(1) was solved numerically to determine $\Omega(q)$.

The perturbation growing rate $\operatorname{Im}(\Omega)$ versus wavevector is shown in Fig.5 for 4-LSM patterns generated at the points $\mathbf{a}$ and $\mathbf{b}$ in Fig.3. The pattern (a) in Fig.5 is obviously stable, i.e. $\operatorname{Im}(\Omega(q))<0$ for $0 \leq q \leq \pi$, while the pattern (b) is unstable $(\operatorname{Im}(\Omega(q))>0$ for some $q$ values). As some individual carrier LSMs composing the pattern are unstable the pattern stability can come from destructive interference between MIs of the carrier LSMs. To qualitatively understand this phenomenon one may imagine the simplified picture: i) perturbation LSMs of different symmetry compose the perturbation state Eq. (8) via four-wave mixing; ii) this state is excited by different (by symmetry) coherent excitations (different carrier LSMs). Hence, whether the perturbation state will be continuously populated depends on the amplitudes of the coherent excitations and on the phase angle(s) between them (the influence of phase angle between the carrier LSMs on the pattern stability is demonstrated in Fig.5). It may happen that at certain combination of the excitation parameters the perturbation state won't be populaed at all. That means the MI-mediated influence of a given carrier LSM on the perturbation (8) can be cancelled by that of the other(s) resulting in the stability of all the carrier LSMs, i.e. the pattern. 


\section{PATTERN FORMATION IN THE PRESENCE OF NOISE}

The stability regions for both 3-LSM and 4-LSM patterns corresponding to the condition $\operatorname{Im}(\Omega)<0$ (damping of the perturbation) are shown by dotted lines in Fig.3. These stability regions were determined numerically via excitation of the pattern starting from a small amplitude seeding wave (S-patterns). Since the stability regions are inside the MI regions of the corresponding excited carrier LSM one might expect that the stable patterns can be spontaneously generated there. This is not always true, however, if the pattern is generated without any seed but in the presence of random noise (N-pattern). When generated from a small amplitude noise the pattern can be unstable because of simultaneous generation of waves with wrong $(\pi / q$ is not integer) wavevectors. The examples of the N-pattern formation are given in Fig.6. Numerical study was performed for 60 particle chain using the standard conservative scheme of numerical integration of the motion equations (1) with cyclic boundary conditions. Random noise of the value between $\pm 0.00005 a$ was generated at each time step and added to the current particles positions. The panels notation in Fig.6 correspond to the points notation in Fig.3. The 4-and 3-LSM patterns generated respectively at the points $\mathbf{a}$ and $\mathbf{c}$ in Fig.3 are really stable and can be attributed to the true spatial patterns since they are similar to those calculated theoretically (compare dotted and solid lines in Fig.4). The pattern at the point $\mathbf{d}$ is inside the 3-LSM stability region and shows periodic modulation in time similar to that of the pattern at the point $\mathbf{b}$ outside the corresponding stability region. Therefore the N-patterns at the points $\mathbf{b}$ and $\mathbf{d}$ can be regarded as spatio-temporal patterns rather than the true spatial patterns.

Spatio-temporal patterns probably also possess certain stability properties though the latter are not so definite as for the spatial patterns. Typical temporal evolution of the spatio-temporal pattern is shown in Fig.7. According to Fig.7 the pattern corresponding to the point $\mathbf{b}$ in Fig.3 is formed over $\sim 30$ periods of vibration and remains rather stable though its contrast slightly decreases in time. Relatively long-time stability of the pattern outside the stability region of the true spatial pattern can be qualitatively understood. Growing up of a perturbation $\delta U_{n}(t)$ in the presence of the unstable pattern at the point $\mathbf{b}$ results in the shift of the quasiharmonic resonance frequency of the optic LSM

$$
\Delta \omega(k=0)^{2}=3 K_{4} \cdot\left\langle\delta U_{n}^{2}(t)\right\rangle_{n, t}
$$

and consequently in the increase of the frequency mismatch $\omega-\omega(k=0)$. Due to this increase the pattern approaches stability region or even enters it and the growing conditions for the perturbation are violated. After damping of the perturbation the pattern returns to the initial point and the process starts again thus giving rise to slow modulation of the pattern amplitude, i.e. results in formation of spatio-temporal pattern. The latter can be stable if the pattern modulation is perfectly periodic, i.e. the modulation amplitude doesn't grow up, otherwise it is obviously unstable. In the long-time scale the evolution of the unstable spatio-temporal pattern due to continues energy collapse can lead to a situation when the vibration amplitude of a particle exceeds the threshold value $U_{t h}^{0}$ what means the point defect formation.

\section{OPTICAL INTENSITY REQUIRED FOR THE PATTERN GENERATION}

Our numerical experiments show that to reach the threshold for the pattern formation with $N_{L S M}=4$ in the L1 lattice the particles in the excited LSM must vibrate with the amplitude $A_{p} \simeq 0.05 a$. The electric field strength $E_{0}$ of the optical excitation at $\omega=1.05 \omega_{0} \simeq 10^{2} \mathrm{~cm}^{-1}$ can be then estimated using motion equation in the harmonic approximation. Suggesting $a=5 \stackrel{\AA}{A}$ and $\gamma=0.05 \omega_{0}$ one obtains $E_{0}=A_{p} m_{p} m_{e}\left[\left(\omega_{0}^{2}-\omega^{2}\right)^{2}+(\gamma \omega)^{2}\right]^{1 / 2} /\left(e_{p} e_{e}\right) \simeq m_{p} / e_{p}$ [V/cm], where $m_{p}$ and $e_{p}$ are the particle mass and charge measured in the free electron units $m_{e}$ and $e_{e}$ respectively. Accordingly, the field strength is of the order of $1 \mathrm{~V} / \mathrm{cm}$ for light particles like electrons and of the order of $10^{5} \mathrm{~V} / \mathrm{cm}$ for ionic solids. The latter value of $E_{0}$ can be reached in the laser pulses. Obviously the pulse duration $T$ must be enough for the pattern to be generated and detected. The generation stage lasts over $30-40$ periods of vibration and $10-20$ periods are probably needed for the pattern detection what in total means $T \simeq 20$ ps. Hence, for the pattern formation in an electronic system (e.g. charge-density wave conductor) the IR laser pulses of energy $W \simeq 10^{-11} m J$ focused into $\sim 0.01 \mathrm{~cm}^{2}$ are required. The pattern formation in an ionic system needs much higher pulse energy $W \simeq 0.1 \mathrm{~mJ}$ unless the smaller focusing area is used.

Thus, in real physical experiment a system will be subjected to external field and examined during a finite time. Therefore it seems reasonable to use some criterion of the pattern relative stability (during finite time period) rather than that given by linear stability analysis. The latter, moreover, seems to be too complicated for spatio-temporal patterns especially for a system with realistic potential. According to the above said the pattern stability regions in Fig.3 must be transformed taking into account the pattern generation and probably detection conditions (see next Section). 


\section{PATTERN GENERATION AND STABILITY FOR $K_{3} \neq 0$}

The 3-LSM pattern solution in this case have the form

$$
\begin{gathered}
U_{n}^{3-L S M}(t)=\frac{1}{2}\left[V_{C 10}+V_{C 11} \exp \left(i \omega t+i \varphi_{11}\right)+V_{C 12} \exp \left(i 2 \omega t+i \varphi_{12}\right)+\right. \\
\cos \left(\frac{\pi}{2} n\right) \cdot\left(V_{C 20}+V_{C 21} \exp \left(i \omega t+i \varphi_{21}\right)+V_{C 22} \exp \left(i 2 \omega t+i \varphi_{22}\right)\right)+ \\
\left.\cos (\pi n) \cdot\left(V_{C 30}+V_{C 31} \exp \left(i \omega t+i \varphi_{31}\right)+V_{C 32} \exp \left(i 2 \omega t+i \varphi_{32}\right)\right)+c . c .\right]
\end{gathered}
$$

In contrast to Eqs (6) and (7) the pattern (9) contains static distortion and also the higher (second) temporal harmonic can not be neglected. Hence the linear stability analysis of the pattern in case $K_{3} \neq 0$ is too complicated. Note, that the approximation of the system with $K_{3} \neq 0$ by a system with effective quartic anharmonicity gives reasonably good results when applied to the carrier LSM stability but not to the pattern stability. According to the estimation given in the previous Section, one can revise the pattern stability conditions and consider the pattern as being relatively stable if it is stable during few tens of periods $T=2 \pi / \omega$. As a new stability criterion one may consider a requirement for a pattern to be stable if it shows well pronounced spatial modulation ( $>10$ per cent) of particle vibration amplitude for a time interval of about 30-50 periods of vibration. The latter time for real system is determined by the pattern detection time and can be even shorter.

The new regions of the pattern relative stability were determined from numerical experiment on the bases of the aforementioned criterion (see shaded regions in Fig.8). The bubbles in Fig.8 show the pattern generation (excited LSM instability) regions for the L1 and L2 lattices (solid and dotted lines, respectively). Dashed-dotted thin lines denote the envelope for the corresponding bubbles and determine the MI cut-off for the excited carrier LSMs. These cut-off lines are determined from the system of equations

$$
\left\{\begin{array}{c}
\Omega(q)=0 \\
\frac{\partial \Omega(q)}{\partial q}=0 \\
E=\sqrt{\left(\omega_{0}^{2}+\frac{3}{4} K_{4} \cdot V_{c 1}^{2}-\omega^{2}\right)^{2}+(\gamma \omega)^{2}}
\end{array}\right.
$$

where $\Omega(q)$ is determined by Eq. (3) and again $K_{4}$ and $\beta$ must be substituted with $K_{4}^{\text {eff }}$ and $\beta^{\text {eff }}$ if $K_{3} \neq 0$. Note, that the stability regions are separated from the envelope lines because in the close vicinity to the latter the perturbation growing rate is too low to form the pattern with well pronounced modulation (at least 10 per cent) during the time of numerical experiment.

It is important to determine the pattern generation and relative stability regions in the $(E, \omega)$ space, i.e. the space of external excitation parameters. They are shown by shaded regions in Fig.9 for 3- and 4-LSM patterns in the L2 lattice with $\gamma=0.025 \cdot \omega_{0}$. The latter value seems quite reasonable for realistic systems. The stability regions were obtained numerically under condition that the pattern shows more than 10 percent modulation in $\left\langle U_{n}^{2}(t)\right\rangle_{t}$ function but doesn't result in the point defect formation (particle jump from its initial minimum in Fig.1a) during 120 periods of vibration. Even in the case of nearly fastest evolution of the 3-LSM pattern it can be considered as stable for $\sim 10$ periods of vibration (see Fig.10) though in the long time scale the system eventually arrives to chaotic state. Taking into account these short-living patterns one may expect the pattern formation phenomenon in rather broad region of the external excitation and system parameters. Note, that the particles vibration in the time interval $3-4(90-120$ periods) in Fig.10 is similar to that in the spatio-temporal pattern in Fig.6c suggesting that the spatio-temporal patterns represent the intermediate state in transition from dynamical order to chaos.

\section{CONCLUSIONS}

The dynamical coherent structure (pattern) formation was studied in the optically driven Klein-Gordon lattice with cubic and quartic anharmonicity in the presence of random noise. It is shown that in general the patterns are characterized by both spatial and temporal modulation of the particles vibration amplitude (so-called spatio-temporal patterns) and can be relatively stable in rather broad region of external excitation and the system parameters.

\section{ACKNOWLEDGMENTS}

This work was supported by Russian Ministry of Science within the program "Fundamental Spectroscopy". 
[1] M.Cross and P.C.Hohenberg, Rev.Mod.Phys. 65, 851 (1993).

[2] F.Melo, P.Umbanhowar, and H.L.Swinney, Phys.Rev.Lett. 72, 172 (1994); ibid 75, 3838 (1995).

[3] P.Umbanhowar, F.Melo, and H.L.Swinney, Nature (London) 382, 793 (1996).

[4] E.Clement et al., Phys.Rev. E 53, 2972 (1996).

[5] L.S.Tsimring and I.S.Aranson, Phys.Rev.Lett. 79, 213 (1997).

[6] B.Denardo, W.Wright, S.Putterman, and A.Larraza, Phys.Rev.Lett. 64, 1518 (1992).

[7] Yuri S. Kivshar, Phys.Rev. B 46, 8652 (1992).

[8] Y.S.Kivshar, O.A.Cubukalo, O.V.Usatenko, D.V.Grinyoff, Int. J. Mod. Phys. B 9, 2963 (1995).

[9] T.Rössler, J.B.Page, Physics Letters A 204, 418 (1995).

[10] Victor M.Burlakov, Phys.Rev.Lett. 80, 3988 (1998).

[11] A.S.Dolgov, Sov.Phys.Sol.State 28, 907 (1986).

[12] A.J.Sievers and S.Takeno, Phys.Rev. Lett. 61, 970 (1988).

[13] J.P.Page, Phys.Rev. B 41, 7835 (1990).

[14] V.M.Burlakov, S.A.Kiselev, and V.N.Pyrkov, Phys.Rev. B 42, 4921 (1990).

[15] T.Rössler and J.B.Page, Phys.Rev.B 51, 11382 (1995).

[16] W.Z.Wang, J.T.Gammel, A.R.Bishop, and M.I.Salkola, Phys.Rev.Lett. 76, 3598 (1996).

[17] T.Rössler and J.B.Page, Phys.Rev.Lett. 78, 1287 (1997).

[18] R.Lai and A.J.Sievers, Phys.Rev.Lett. 81, 1937 (1998).

[19] A.Tsurui, Progr.Theor.Phys. 48, 1196 (1972).

[20] V.M.Burlakov, S.A.Kiselev, Sov.Phys.JETP 72, 854 (1991).

[21] Y.S.Kivshar, M.Peyrard, Phys.Rev. A 46, 3198 (1992).

[22] Yu.S.Kivshar, Phys.Rev. E 48, 4132 (1993).

[23] K.W.Sanduski and J.B.Page, Phys.Rev. B 50, 866 (1994).

[24] V.M.Burlakov, S.A.Darmanyan, and V.N.Pyrkov, Sov.Phys.JETP 108, 904 (1995).

[25] V.M.Burlakov, S.A.Darmanyan, and V.N.Pyrkov, Phys.Rev. B 54, 3257 (1996). 


\section{Figure captures}

Fig.1. a) Incite potential function for a particle in the Klein-Gordon L1- (1) and L2 (2) lattices (see the text).

b) $V_{C j} \propto E_{0}$ plot (see Eq. 2 in the text). Curves 1, 2 and 3 correspond to $j=1$ (first harmonic), 2 (second harmonic) and 0 (static displacement or, zero harmonic) respectively. Lines correspond to potential of the L1 lattice and symbols to that of the L2 lattice. The external field frequency is $\omega=0.9 \omega_{0}$ (solid lines) and $\omega=1.05 \omega_{0}$ (dotted lines), $\gamma=0.025 \omega_{0}$.

Fig.2. Perturbation relative growing rate (relative increment) $\operatorname{Im}(\Omega(q) / \omega)$ for $\omega=1.05 \omega_{0}$ and $\gamma=0.025 \omega_{0}$ in the L1- (a) and L2 (b) lattices; (c) MI of the free $\left(\gamma=0, E_{0}=0\right)$ nonlinear LSM with $k=0$ in the lattice L2. External field amplitude $E_{0}$ was chosen for panels a) and b) in a way when the $V_{C 1}$ value is equal to $0.14(1), 0.1$ (2) and 0.07 (3). Curves (4)-(6) in the panel (b) were calculated from Eq. (3) for the lattice of L1-type with effective quartic anharmonicity $K_{4}^{\text {eff }}=K_{4} \cdot\left(1+\left(\frac{K_{3}}{\omega_{0}^{2}}\right)^{2} \cdot V_{C 1}^{2}\right)-\frac{11}{12}\left(\frac{K_{3}}{\omega_{0}}\right)^{2}$ and $\beta^{\text {eff }}=\frac{7}{12} K_{4} V_{C 1}^{2}$ (see text). The $E_{0}$ values for curves (4), (5) and (6) in the panel b) are equal to those corresponding to $V_{C 1}$ values for curves (1), (2) and (3) respectively.

Fig.3. Carrier LSM modulation instability regions in the $\left(E_{0}, \gamma\right)$ space enclosed by solid curves and corresponding to the pattern generation by external field with $\omega=1.1 \omega_{0}$ (3-LSM patterns) and with $\omega=1.05 \omega_{0}$ (4-LSM patterns) in the L1 lattice. The calculated pattern stability regions are enclosed by dotted curves.

Fig.4. The stable 3-LSM and 4-LSM patterns (the $\left\langle U_{n}^{2}(t)>_{t}\right.$ function) calculated (solid lines) and obtained by numerical simulation in the presence of noise (dotted lines) at the points $\mathbf{a}$ (a) and $\mathbf{b}$ (b) in Fig.3. Thin dashed lines show the amplitude square of the carrier LSM prior the pattern formation has began.

Fig.5. Relative increment $\operatorname{Im}(\Omega(q) / \omega)$ calculated for stable (1) and unstable (2) 4-LSM patterns (7) corresponding to the points $\mathbf{a}$ and $\mathbf{b}$ in Fig.3. Dotted line shows the $\operatorname{Im}(\Omega(q) / \omega)$ function calculated with the same parameters as (1) but with $\phi_{1}$ substituted with $\phi_{1}+\pi / 4$.

Fig.6a-d. Molecular dynamic simulation of the N-pattern (see text) formation in 60-particle L1 lattice with cyclic boundary conditions for $\gamma$ and $E_{0}$ corresponding to the points a-d respectively in Fig.3. Particle displacements from corresponding equilibrium positions are multiplied by factor 3 for (a) and (b) and by factor 2 for (c) and (d).

Fig.7. The $\left\langle U_{n}^{2}(t)\right\rangle_{t}$ pattern obtained from numerical experiment with L1 lattice at the point $\mathbf{b}$ in Fig.3 and taken after 25 (1), 50 (2), 75 (3), and 100 (4) periods of external field vibration. The time average was taken over 10 periods.

Fig.8. The pattern generation and relative stability regions (shaded) for the L1 lattice (light grey) and the L2 lattice (dark grey) for $\omega=1.05 \omega_{0}$. The bubbles enclose the 5-, 4- and 3-LSM pattern generation regions in the L1(light grey) and L2 (dark grey) lattices. Dashed lines denote the envelope for the corresponding bubbles.

Fig.9. The pattern generation and relative stability regions (shaded) for the L2 lattice with $K_{2}=30000, \gamma=$ $0.025 \omega_{0}$. Solid lines enclose the pattern generation regions determined via numerical simulation for the L2 lattice while dotted lines enclose those obtained from equations $\Omega(q=\pi / 3)=0$ and $\Omega(q=\pi / 2)=0$ for the L1 lattice with effective $K_{4}$ and $\beta$ (see capture to Fig.2). In the calculations the threshold value for the carrier LSM amplitude was taken equal to $\simeq 0.1 \cdot a$. Solid symbol denotes the point at which the pattern formation process is presented in Fig.10.

Fig.10. a) Molecular dynamic simulation of the N-pattern formation in the 60-particle L2 lattice for external field parameters corresponding to the point shown in Fig.9. Particle displacements from corresponding equilibrium positions are multiplied by factor 3. Vertical dotted lines denote the starting time moments of the $\left\langle U_{n}^{2}(t)\right\rangle_{t}$ calculation shown in b). The averaging was taken over 10 periods. 

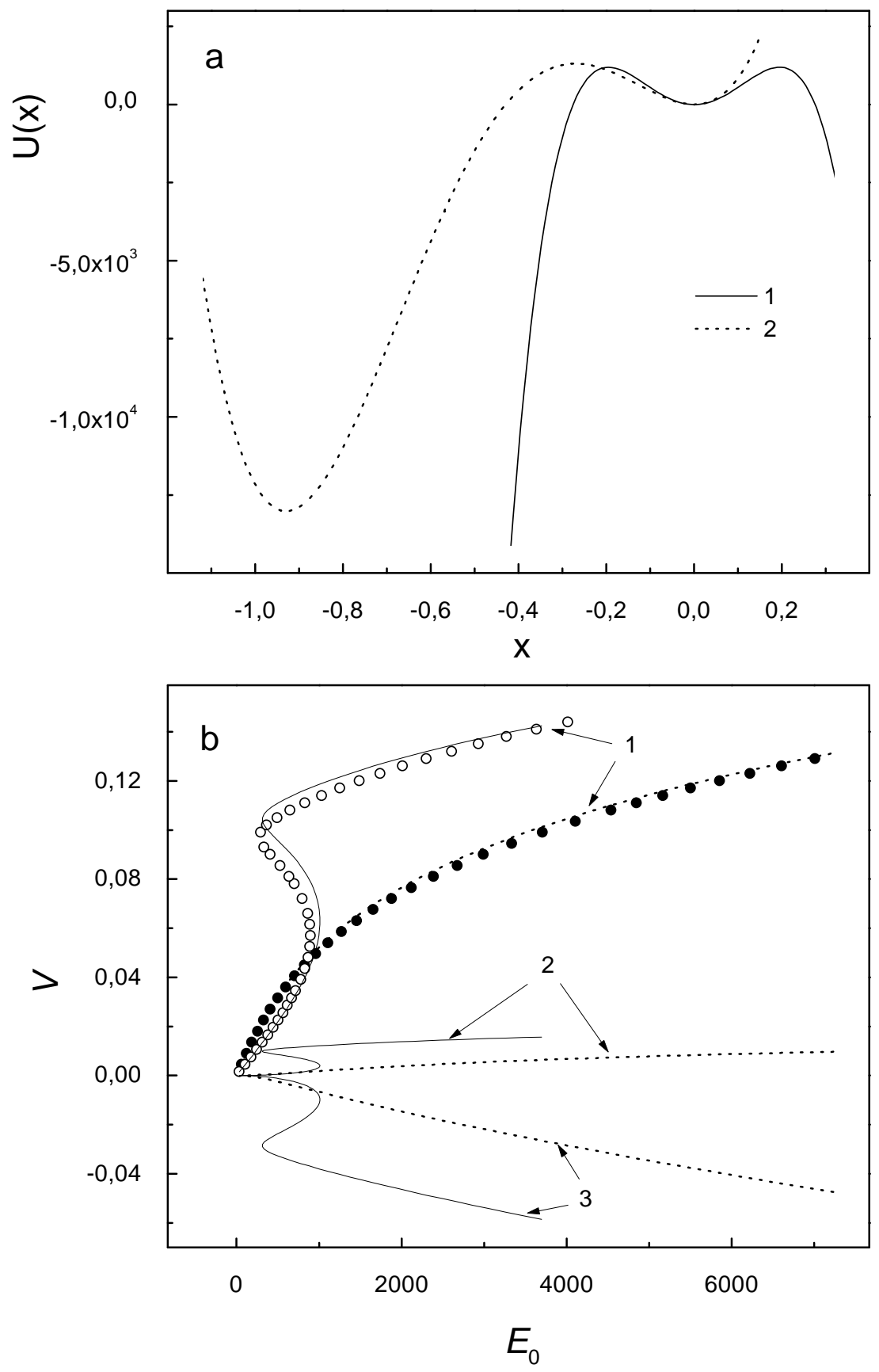

Fig.1. Victor M. Burlakov, Spatial- and spatio-temporal pattern formation in optically driven discrete systems 


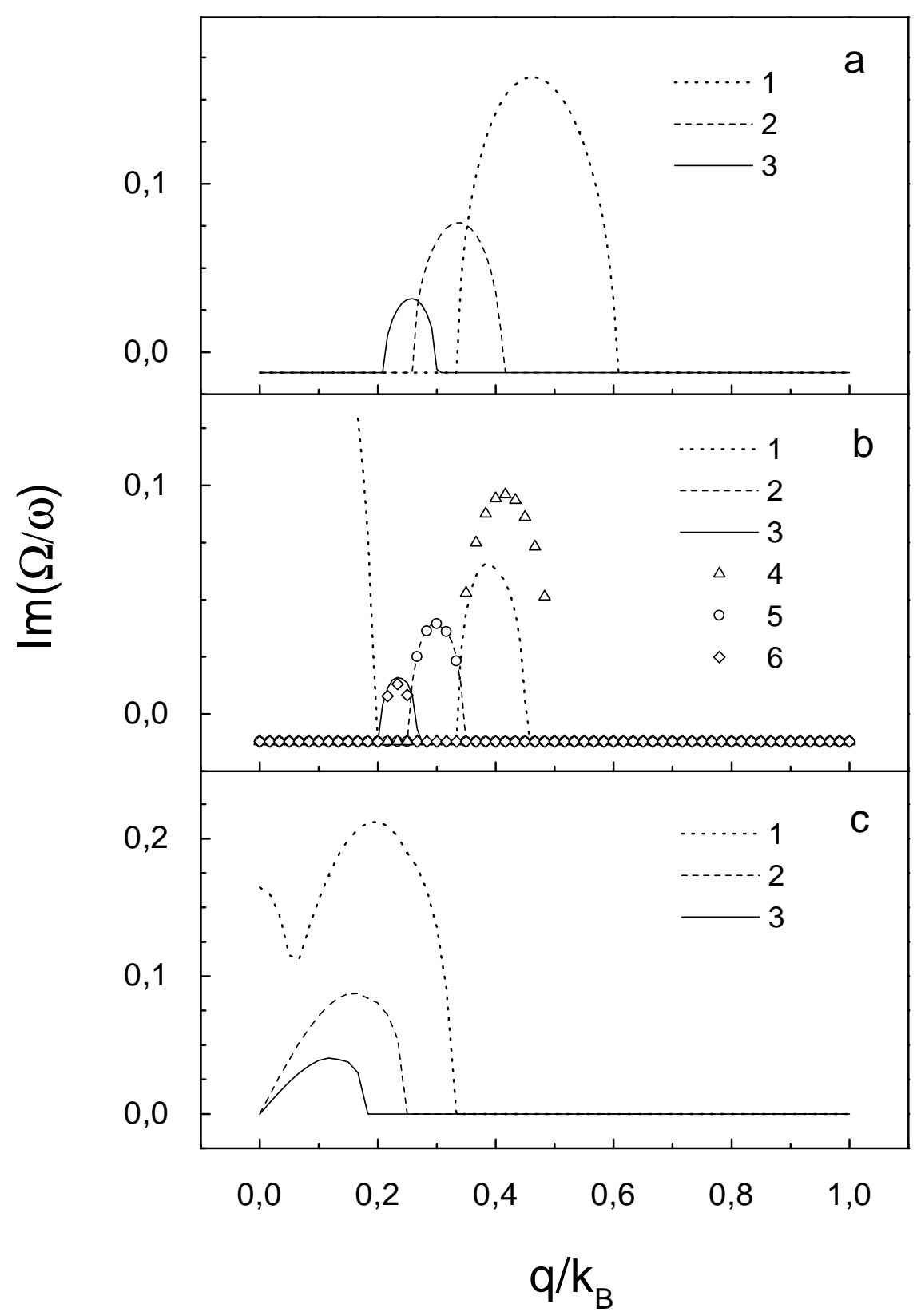

Fig.2. Victor M. Burlakov, Spatial- and spatio-temporal pattern formation in optically driven discrete systems 


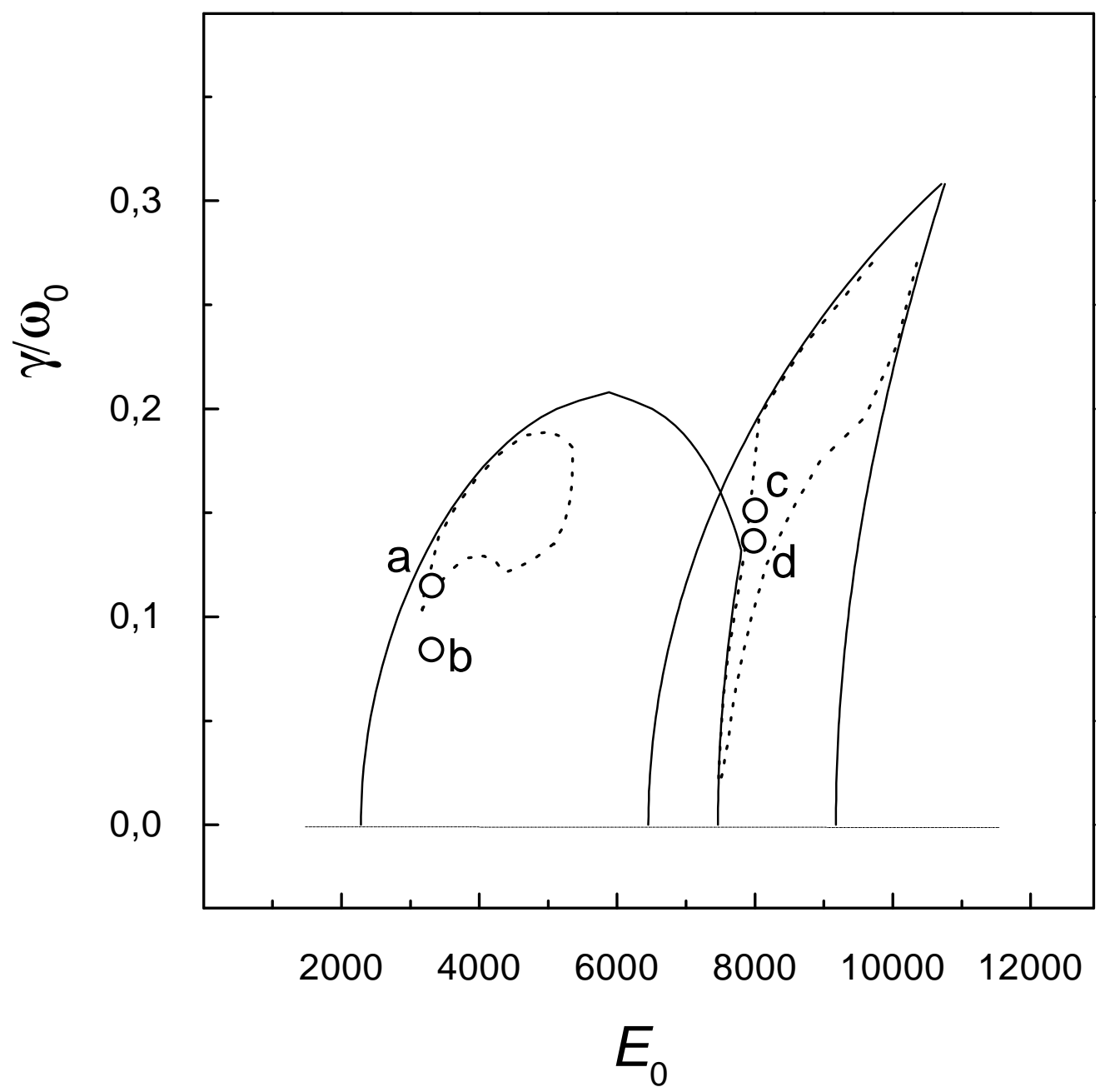

Fig.3. Victor M. Burlakov, Spatial- and spatio-temporal pattern formation in optically driven discrete systems 


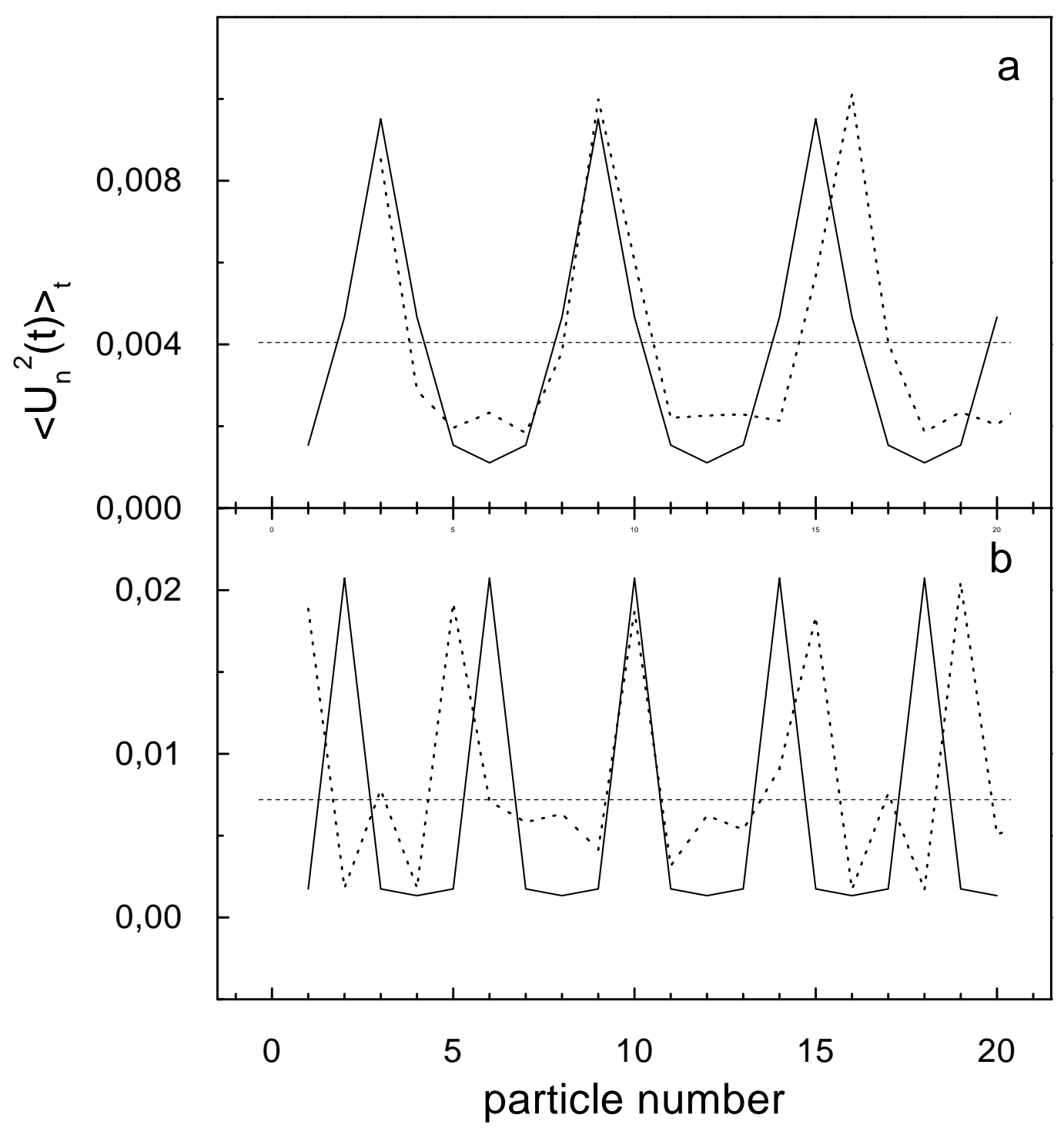

Fig.4. Victor M. Burlakov, Spatial- and spatio-temporal pattern formation in optically driven discrete systems 


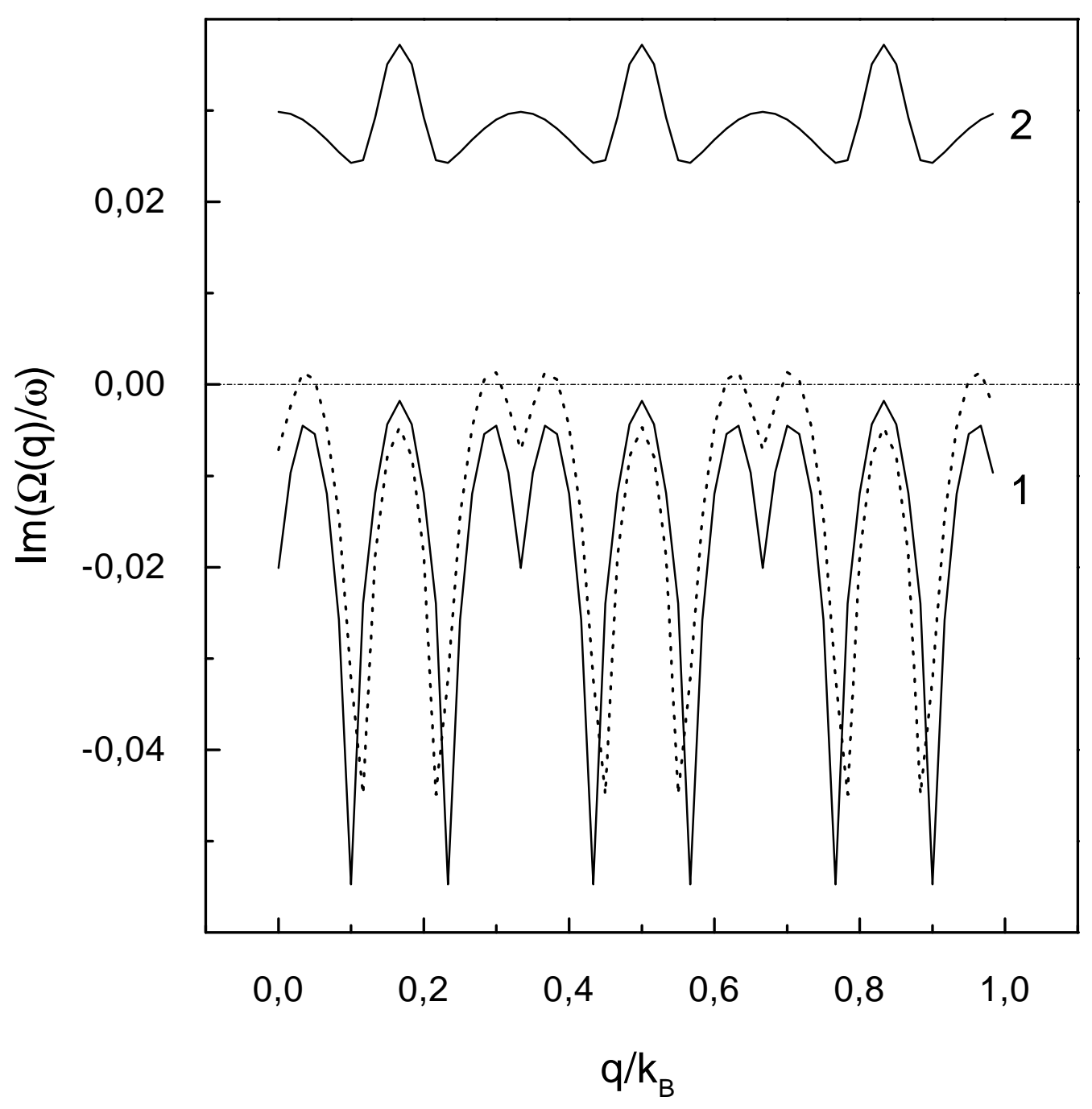

Fig.5. Victor M. Burlakov, Spatial- and spatio-temporal pattern formation in optically driven discrete systems 


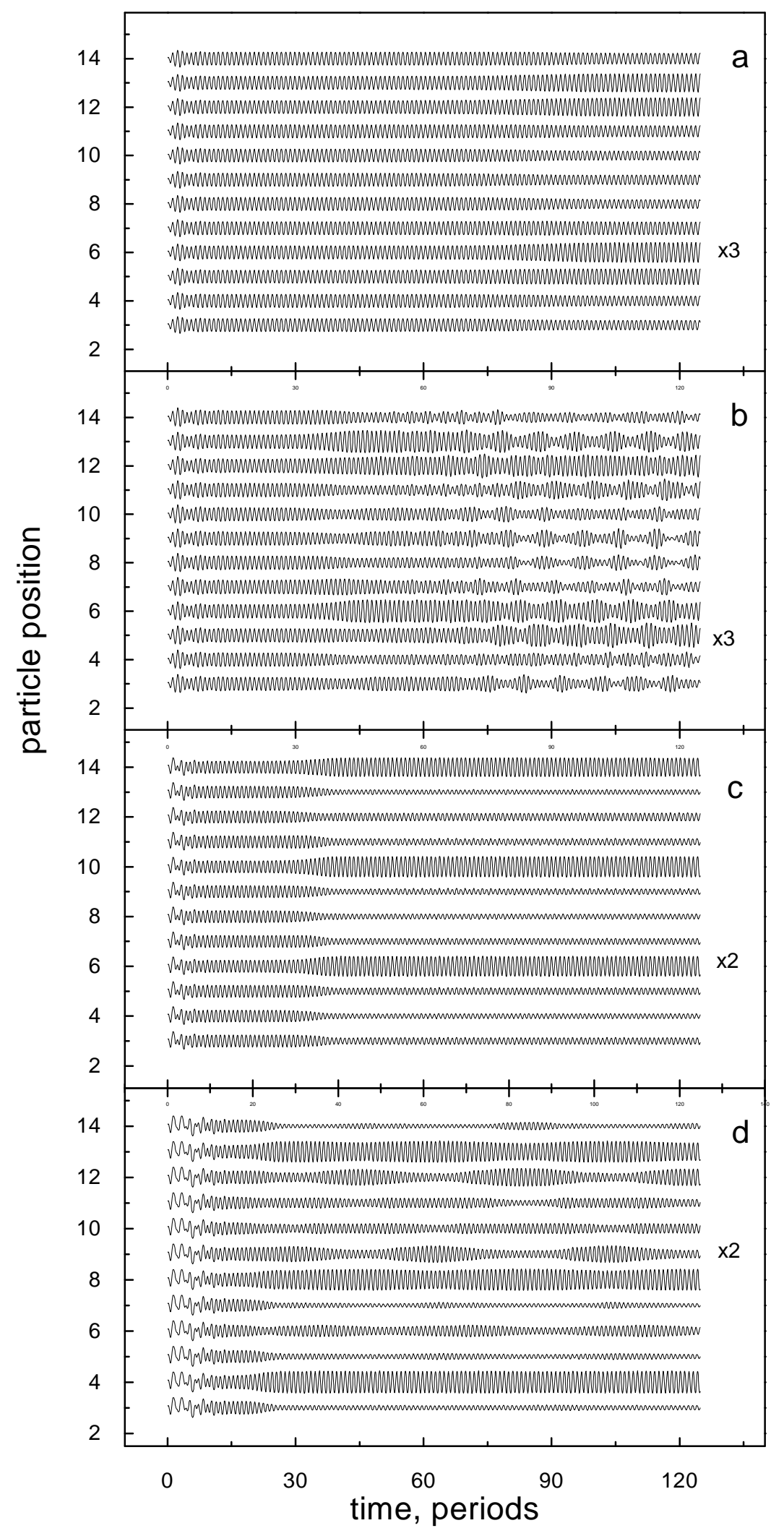

Fig.6. Victor M. Burlakov, Spatial- and spatio-temporal pattern formation in optically driven discrete systems 


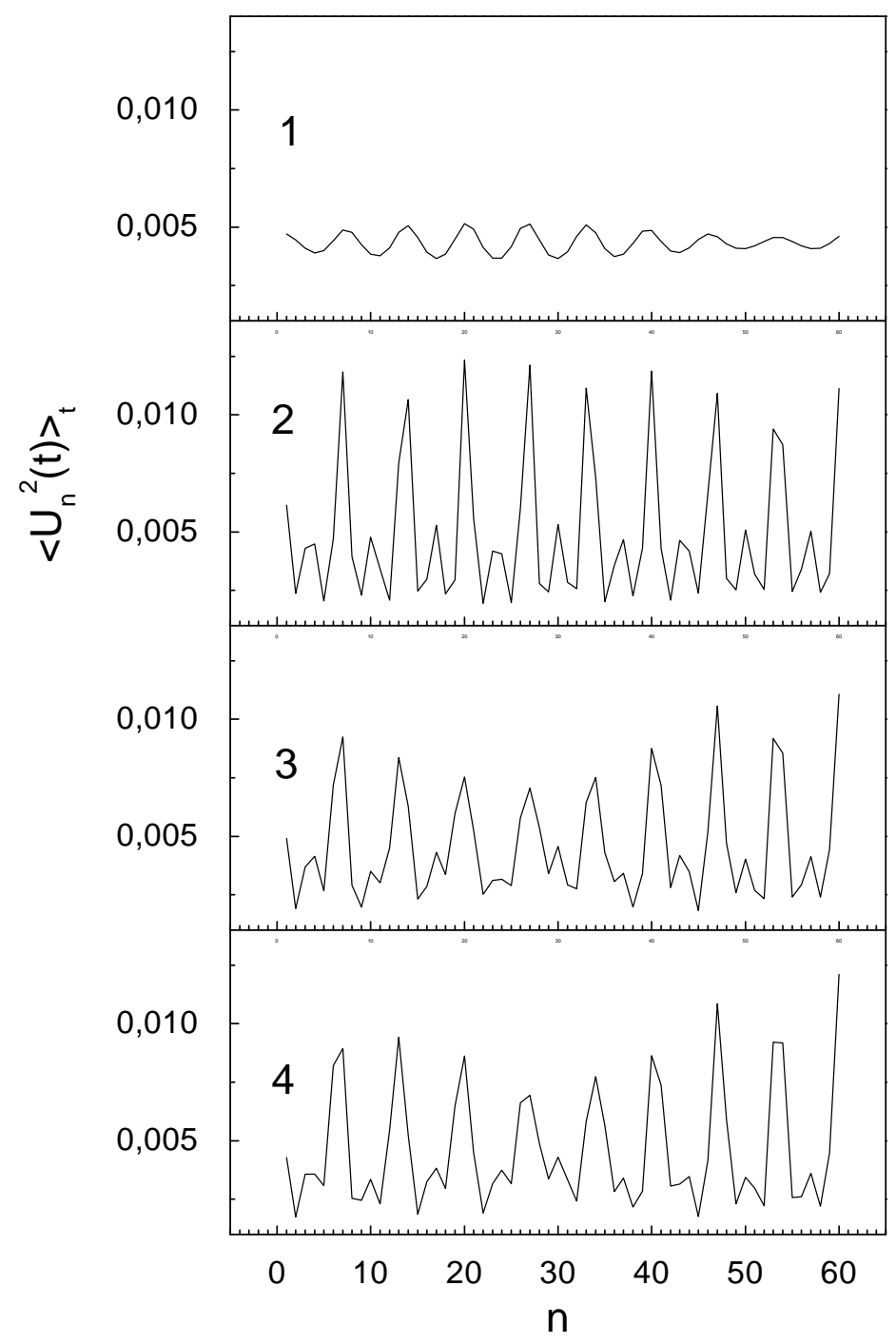

Fig.7. Victor M. Burlakov, Spatial- and spatio-temporal pattern formation in optically driven discrete systems 


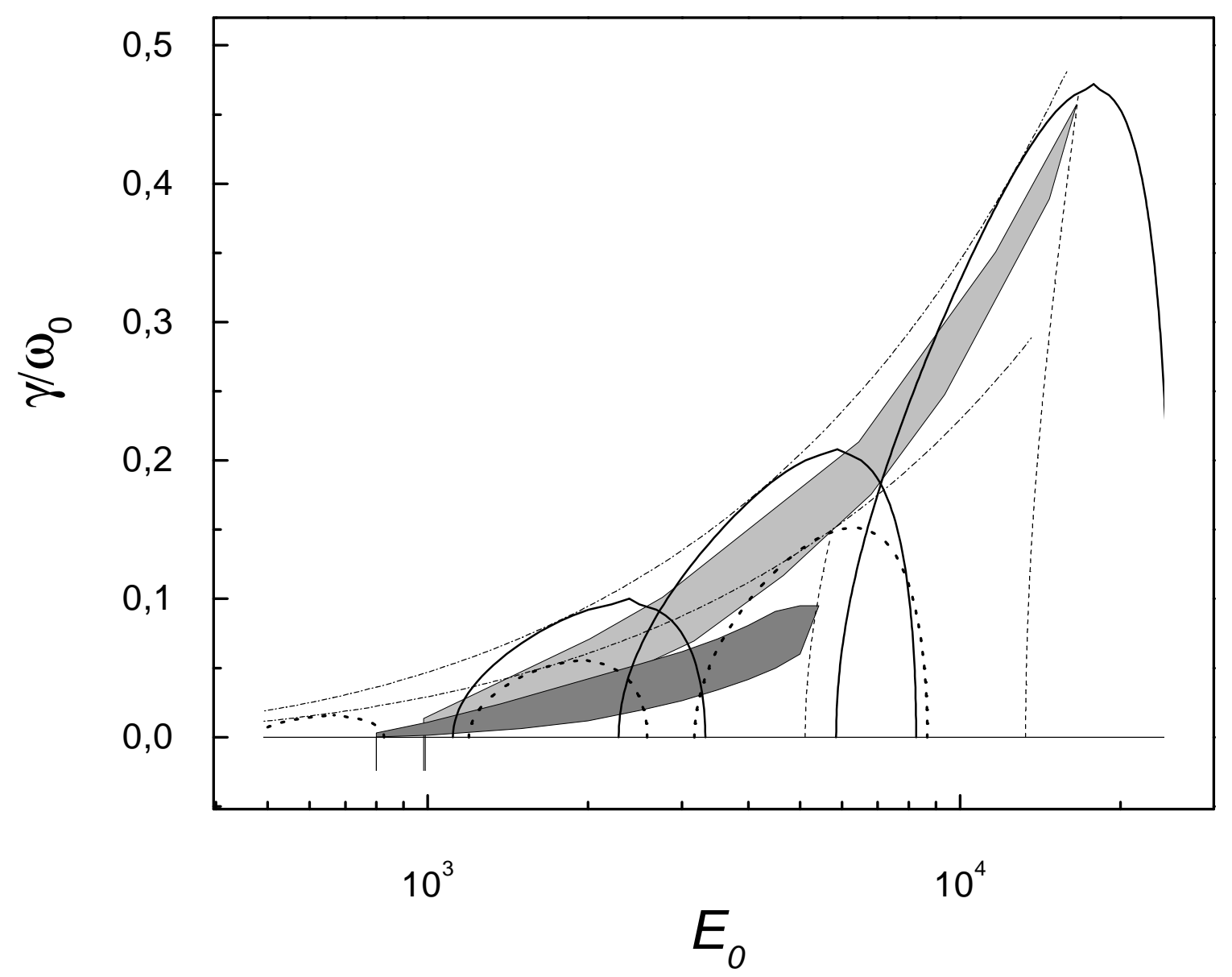

Fig.8. Victor M. Burlakov, Spatial- and spatio-temporal pattern formation in optically driven discrete systems 


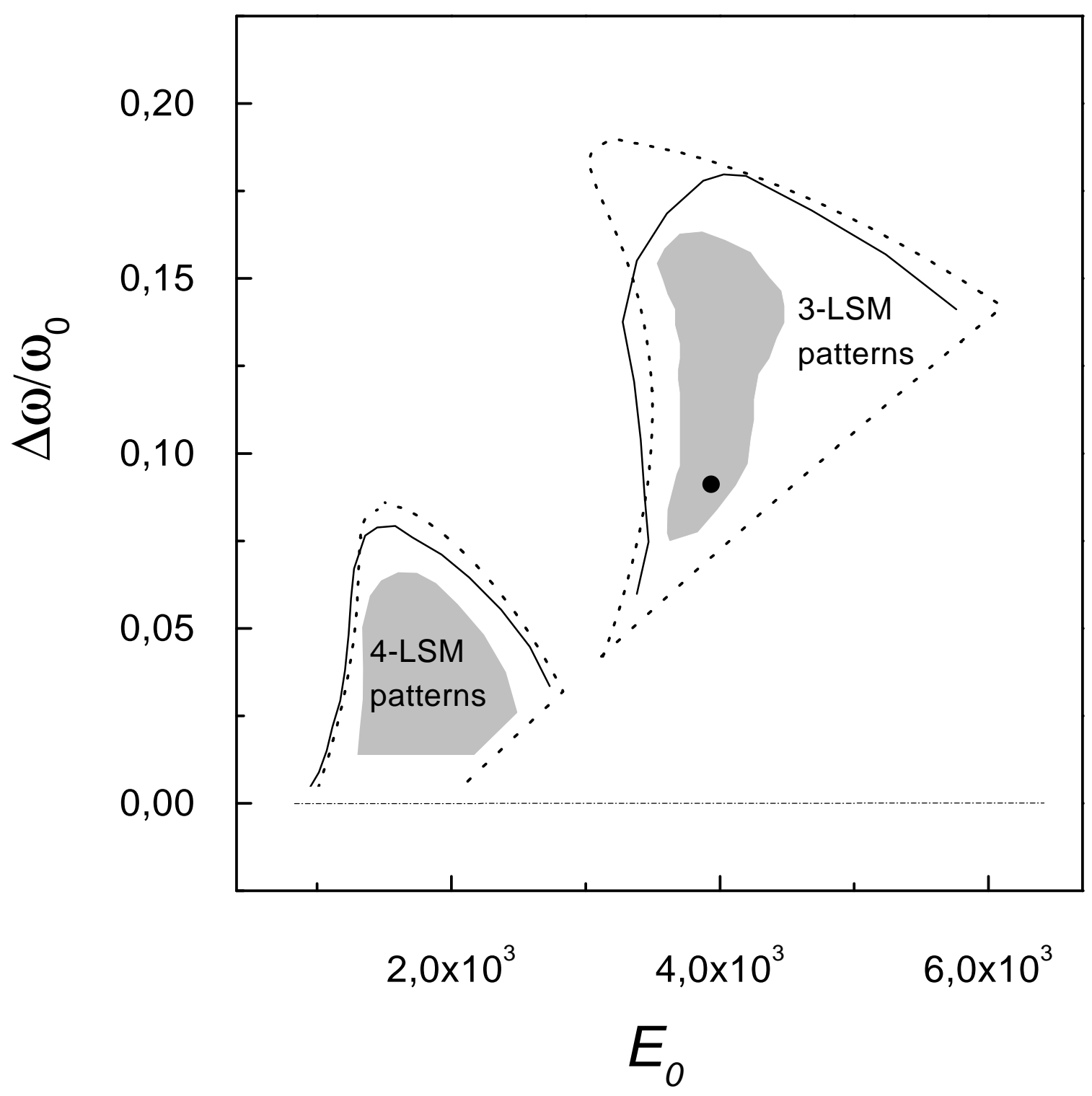

Fig.9. Victor M. Burlakov, Spatial- and spatio-temporal pattern formation in optically driven discrete systems 

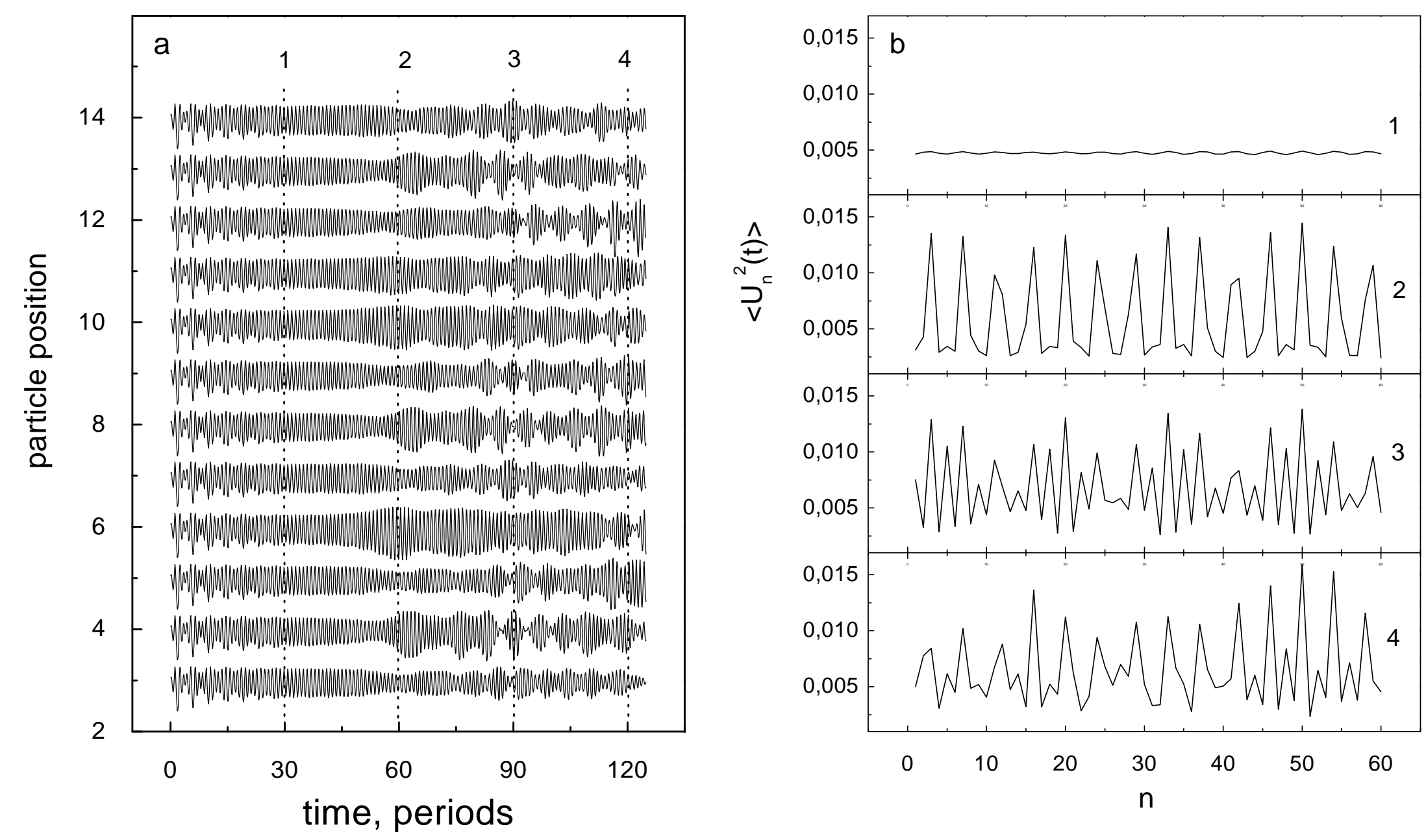

Fig.10. Victor M. Burlakov, Spatial- and spatio-temporal pattern formation in optically driven discrete systems 